\title{
MÉTAPSYCHOLOGIE, ÉPISTÉMOLOGIE ET ÉTHIQUE DE LA CLINIQUE PSYCHANALYTIQUE
}

Hélène Tessier ${ }^{*}$

RÉSUMÉ. Cet article traite de la différence entre la psychotérapie et la psychanalyse et analyse les objectifs respectifs des deux méthodes, en se référant à la théorie de la séduction généralisée de Jean Laplanche, ansi qu'au projet d'émancipation que cette théorie confère à la psychanalyse. Il fait valoir qu'il existe un rapport très étroit entre la théorie et la pratique en psychacanalyse, cela signifie que la pratique implique toujours le choix d'un modèle métapsychologique. L'article soutient par ailleurs que tous les modèles ne sont pas équivalents quant à leur conception de la transformation, objectif inhérent tout-àfait à la pratique de la psychanalyse.

Mots clés: Psychanalyse clinique; théorie de la séduction génèralisée; métapsychologie.

\section{METAPSICOLOGIA, EPISTEMOLOGIA E ÉTICA DA CLÍNICA PSICANALÍTICA}

RESUMO. O presente artigo analisa as diferenças entre psicoterapia e psicanálise e situa o quadro e os respectivos objetivos do seus métodos de acordo com os principios da teoria da sedução generalizada de Jean Laplanche. Considera também, e como destaque desta teoria, a visão de emancipação que lhe é atribuída à psicanálise. Com esses fundamentos, o artigo sustenta que existem laços estreitos entre teoria e prática, que a prática da psicanálise implica na escolha de um modelo metapsicológico e que todos os modelos não podem ser tomados como equivalentes, considerando o objetivo de transformação inerente à prática psicanalítica dos nossos dias.

Palavras-chave: Psicanálise clínica; teoria da sedução generalizada; metapsicolologia.

\section{METAPSYCHOLOGY, EPISTEMOLOGY AND ETHICS OF CLINICAL PSYCHOANALYSIS}

ABSTRACT. This paper discusses the differences between psychotherapy and psychoanalysis, and places the framework and objectives of their respective methods in accordance with the principles of the Jean Laplanche's theory of generalized seduction, as well as the project of emancipation that this theory confers to psychoanalysis. With these foundations, it argues that there are close relationships between theory and practice, that the practice of psychoanalysis always implies the choice of a metapsychological model, and that all models cannot be taken as equivalent, considering their goal of transformation, an aim which is inherent to psychoanalytic practice today.

Key words: Psychoanalysis practice; theory of generalized seduction; metapsychology.

\section{METAPSICOLOGÍA, EPISTEMOLOGÍA Y ÉTICA DE LA CLÍNICA PSICOANALÍTICA}

RESUMEN. El presente artículo analiza las diferencias entre psicoterapia y psicoanálisis y sitúa el cuadro y los respectivos objetivos de sus métodos de acuerdo con los principios de la teoría de la seducción generalizada de Jean Laplanche. Considera también, y como destaque de esta teoría, la visión de emancipación que le es atribuida al psicoanálisis. Con estos fundamentos, el artículo sostiene que existen lazos estrechos entre teoría y práctica, que la práctica del psicoanálisis implica en la elección de un modelo metapsicológico y que todos los modelos no pueden ser tomados como equivalentes, considerando el objetivo de transformación inherente a la práctica psicoanalítica de nuestros días

Palabras-clave: Psicoanálisis clínico; teoría de la seducción generalizada; metapsicología.

Professeure agrégée à l'Université Saint-Paul, Otawa, Canadá. Directrice du Centre de recherche sur le conflit (Université Saint-Paul). Avocate et psychanalyste. 
Il existe des liens étroits entre théorie et pratique en psychanalyse. La pluralité des orientations psychanalytiques et l'éclectisme avec lequel les psychanalystes rendent compte de leur pratique soulèvent par ailleurs la question du choix entre les différentes orientations théoriques. Wallerstein (1988) soutient par exemple que le pluralisme dans la théorie comporte peu d'incidences, puisque les psychanalystes se reconnaitraient facilement dans une pratique clinique unifiée. $\mathrm{La}$ théorie n'aurait-elle pas d'influence sur la pratique ? Est-ce exact? Est-ce même possible?

Par ailleurs, les nombreuses théories psychanalytiques peuvent-elles simplement coexister, y compris quand elles supposent des définitions incompatibles des principaux concepts de la psychanalyse? Si c'est le cas, que révèlerait cette coexistence sur la pertinence de la métapsychologie? Le discrédit dans laquelle on la tient maintenant seraitil mérité?

Je me propose d'examiner trois questions:

1. Existe-t-il un lien entre métapsychologie, théorie psychanalytique et pratique?

2. Si oui, doit-on obligatoirement effectuer un choix entre les différentes orientations théoriques?

3. Si tel est le cas, sur quels critères peut-on baser ce choix?

\section{LE LIEN ENTRE LA THÉORIE ET LA PRATIQUE : UNE THÉORIE DE LA TRANSFORMATION}

Les rapports entre la théorie et la pratique en psychanalyse sont depuis longtemps une source d'interrogations. Les écrits techniques de Freud, bien que donnant des indications sur la méthode analytique, ne comportent pas de liens évidents avec ses écrits sur la métapsychologie. La date de rédaction de ces écrits est d'ailleurs antérieure à celle des écrits sur la première topique et, bien sûr, à celle des divers remaniements métapsychologiques qui leur ont succédé. De la même façon, L'interprétation du rêve, qui constitue un texte important sur la technique de la libre association, est arrivé très tôt dans l'œuvre de Freud (1900/1992). Cette chronologie montre que la découverte de la méthode psychanalytique représente un pas décisif dans l'élaboration métapsychologique. Cette idée est confirmée dans la définition que Freud donne de la psychanalyse: pour lui, il s'agit en premier lieu d'un procédé d'investigation de processus psychiques, difficilement accessibles autrement.

Il est bien connu que la découverte de la psychanalyse a été avant tout la découverte d'une méthode psychothérapeutique, dérivée de la méthode cathartique. Ce qui frappe aujourd'hui dans la psychanalyse contemporaine, notamment dans les comptes-rendus cliniques, c'est que la méthode psychanalytique semble perdre sa spécificité. Une telle tendance correspond aussi à un mouvement dans la théorie: la théorie psychanalytique se distingue de moins en moins d'autres théories psychologiques. On le constate notamment le cas des courants relationnels et dans le rôle qu'ils attribuent aux théories de l'attachement. Par ailleurs, évoquer, sans plus de précision, la théorie psychanalytique constitue maintenant une impossibilité. La quantité d'orientations théoriques et les divergences qu'elles comportent ne permettent plus de croire que la théorie psychanalytique renvoie à une réalité commune. De plus, les théories psychanalytiques sont le plus souvent incompatibles et elles s'appuient sur des postulats épistémologiques qui, fréquemment, s'opposent Une telle situation contribue à creuser l'écart entre théorie psychanalytique et réalité pratique.

Les rapports entre théorie et pratique constituent la question centrale de la psychanalyse: celle-ci n'est pas une discipline théorique, mais une théorie de la pratique. La psychanalyse est indissociable d'une visée de transformation. Quand Freud (1937/1998) classe la psychanalyse, avec l'enseignement et le gouvernement, dans la catégorie des trois métiers impossibles, il met concrètement en évidence le caractère central de l'objectif de changement qui définit ces champs d'activité.

On pourrait immédiatement m'objecter que la psychothérapie en général vise le changement et que cette caractéristique ne distingue absolument pas la psychanalyse des autres formes d'intervention thérapeutique. S'il est vrai que toute intervention thérapeutique vise le changement, on peut dire que la psychanalyse vise une forme particulière de transformation. Freud (1923/1981) la décrit sous la forme de l'accession à la liberté, à une forme liberté de choix: "la tâche de celle-ci (la psychanalyse) n'est pas de rendre impossible les réactions morbides, mais d'offrir au moi du malade la liberté de décider pour ceci ou pour cela" (p. 265). Ainsi, dans des conditions favorables, la liberté, ou une liberté accrue, constitue, selon Freud, l'issue de la cure psychanalytique. Il faut donc en conclure que la psychanalyse exercerait une action libératrice. Corollairement, la nécessité d'une libération suppose l'idée d'une aliénation préalable. L'idée d'une force aliénante qui agit dans l'âme a pris chez Freud la forme d'un inconscient dynamique et conflictuel. Malgré les différents remaniements et fourvoiements que la notion d'inconscient a connus dans son oeuvre (Laplanche, 1999, 2006), Freud est resté fidèle à l'idée de l'inconscient en tant que force contraignante, qui 
apparaît à l'individu comme lui étant étrangère, qui fait que le moi n'est pas maître chez lui, que l'homme, même sous l'effet des meilleurs conseils et de la plus grande bonne volonté, n'agit pas nécessairement en fonction de ce qu'il pense vouloir. Dans ses rapports avec l'inconscient, la cure psychanalytique aurait non seulement une visée de transformation, mais aussi une visée d'émancipation. Il s'agit en effet d'appréhender cette rationalité étrangère au sujet, en espérant que celui-ci sera alors plus libre de fonder son action sur des éléments qui ne relèvent plus d'une contrainte dont la source lui échappe.

C'est ici qu'intervient la question du choix d'une théorie métapsychologique. Il n'est pas évident que toutes les théories psychanalytiques soient compatibles avec la conviction que la psychanalyse comporte, dans son essence, une visée d'émancipation. Cette visée d'émancipation ne se limite pas à la cure elle-même. La théorie psychanalytique est aussi une conception de l'âme humaine. Pour être conciliable avec l'hypothèse d'un projet de transformation, cette conception doit décrire une âme capable de se transformer. Par ailleurs, pour satisfaire aux conditions de possibilité de l'émancipation, elle doit reposer sur des principes propres à en sauvegarder l'autonomie. En d'autres termes, elle ne peut, d'une part, inclure une conception normative du développement, de la normalité, de la pathologie. Elle doit être attentive aux dangers de la suggestion et à celui de la substitution des positions de l'analyste en lieu et place de celles de l'analysant. D'autre part, l'idée même d'émancipation implique la conviction qu'en dehors de toute considération empirique, il existe pour l'humain, une forme de vie émancipée, ontologiquement supérieure à l'état d'aliénation qui caractérise sa situation présente. La théorie métapsychologique doit ainsi répondre à deux critères en apparence contradictoire: les conditions de sauvegarde d'une autonomie tout à fait singulière, respectueuse de l'histoire individuelle de chacun et, en même temps, le postulat d'une forme de vie d'âme essentiellement meilleure, plus libre, à laquelle chacun est en droit d'aspirer.

Ces considérations revêtent peut-être un aspect extrêmement théorique, mais elles sont en réalité concrètes et peuvent servir de grille d'analyse aux théories de la pratique psychanalytique que nous connaissons. Je voudrais donc voudrais examiner les trois points suivants:

1. Les rapports entre métapsychologie et théorie de la pratique.

2. Quelques aspects des théories actuelles sur la pratique en psychanalyse.
3. En conclusion, la dimension éthique des choix épistémologiques.

\section{LES RAPPORTS ENTRE MÉTAPSYCHOLOGIE ET PRATIQUE PSYCHANALYTIQUE}

Avant de continuer notre investigation, il faut s'entendre sur un point qui ne fait pas l'unanimité parmi les psychanalystes et qui repose lui-même sur un choix épistémologique. Il s'agit des rapports entre psychanalyse et psychothérapie. Le développement que je viens de faire sur la visée d'émancipation est, à mon avis, spécifique à la psychanalyse. Il faut cependant expliquer dans quel contexte.

Quelle différence y a-t-il entre psychanalyse et psychothérapie? Je reprends sur ce point la position de Laplanche (2007) pour qui psychanalyse et psychothérapie constituent deux réalités à la fois distinctes et interreliées. Pour Laplanche, l'analyse au sens strict ne peut se concevoir en dehors de la situation et de la méthode analytiques, sur laquelle nous reviendrons un peu plus loin. Le travail d'analyse est analytique, au sens étymologique du terme, c'est à dire fondé sur la déliaison. Il se distingue du travail de synthèse qui caractérise le travail psychothérapeutique. Par ailleurs, comme le souligne Laplanche, la cure psychanalytique ne comporte qu'une petite partie de travail strictement analytique: les interventions psychothérapeutiques occupent la plus grande partie d'une analyse, y compris dans le cas purement hypothétique où cette cure se déroulerait de façon rigoureusement classique - si tant est qu'une telle chose existe. Je l'ai déjà dit, mais il est important de le rappeler ici: la distinction effectuée par Laplanche comporte déjà une référence à un modèle métapsychologique. Nous décrirons brièvement ce modèle plus loin. Revenons pour le moment aux distinctions entre psychanalyse et psychothérapie, sans quoi la discussion sur les liens entre théorie et pratique risque de mener à la confusion. Selon Laplanche, donc, seul l'acte psychanalytique, en tant que travail de déliaison, porte sur les dérivés des processus inconscients. Les autres procédés psychothérapeutiques, qu'ils se produisent dans le cadre d'une psychanalyse ou dans celui d'une psychothérapie $^{1}$, mettent en jeu le sujet tel que constitué. Dans cette perspective, la psychothérapie se distingue de la psychanalyse par le rôle qu'y joue la

1 Le terme psychothérapie se réfère ici aux psychothérapies de type analytique, mais aussi aux psychothérapies de soutien fondées sur l'écoute et l'autostructuration du patient. Il exclut cependant les thérapies de type cognitivobehavioral, qui impliquent des interventions et des objectifs différents. 
synthèse, sous l'aspect d'une remise en forme narrative, de constructions et de reconstructions, voire de subjectivation. Ces derniers procédés relèvent de la fonction psychothérapeutique et, bien qu'on les retrouve en psychanalyse, ils ne servent pas à la définir.

Insistons cependant sur le fait que la distinction entre psychothérapie et psychanalyse ne conduit pas à discréditer la psychothérapie. Laplanche souligne au contraire que la psychothérapie permet des progrès importants, notamment en ce qui a trait à l'autohistorisation, et que, dans des conditions favorables, elle occasionne un mieux être significatif. La question des indications d'analyse entre aussi en jeu: il est loin d'être certain que le travail de déliaison qu'implique l'analyse soit toujours approprié. Établir une distinction entre psychanalyse et psychothérapie permet de mieux situer leur action respective sur les plans topique et métapsychologique.

À partir du moment où l'on a établi une différence entre psychanalyse et psychothérapie, on peut examiner de façon plus précise les liens entre théorie et pratique en psychanalyse, puisque nous avons défini à quoi se réfère la psychanalyse dans notre hypothèse de travail. Celle-ci, dans la définition stricte proposée par Laplanche, se caractérise par un travail de déliaison et porte sur les manifestations ou sur les dérivés de l'inconscient, ainsi que sur les processus défensifs. Cette position implique, d'une part 1) une définition de l'inconscient et d'autre part; 2) une description des conditions méthodologiques qui permettent d'accéder à ses dérivés.

Quant à la définition de l'inconscient, je ne dirai que quelques mots sur la théorie de la séduction généralisée et sur l'hypothèse traductive du refoulement. Celles-ci comportent des considérations sur les origines de l'inconscient, sur sa nature essentiellement sexuelle, sur le caractère singulier de ses contenus et sur son mode de fonctionnement (Laplanche 1997, 1999, 2006). Je mentionnerai seulement que la sexualité à laquelle se réfère la théorie de la séduction généralisée, sous l'angle de l'inconscient sexuel, n'est pas la sexualité au sens courant du terme, mais la sexualité infantile, perverse et polymorphe, qui envahit l'ensemble des activités humaines y compris les fonctions d'autoconservation $^{2}$. Cette sexualité est auto-érotique. Elle est toujours à la recherche d'excitation et fonctionne selon une logique de décharge. De ce fait les pulsions

2 La théorie de la séduction généralisée se réfère aussi à la sexualité narcissique, sous l'angle des pulsions sexuelles de vie. Celles-ci permettent le travail de liaison opéré par le moi et sont en effet sollicitées par le travail psychothérapeutique. sexuelles agissent sur le mode de l'attaqueet elles attaquent le moi de l'intérieur. Il s'agit d'une attaque contre les tentatives de liaison du moi. Les pulsions sexuelles coupent l'affect de ses représentations. Dans leur aspect délié, elles se font connaître sous la forme de l'angoisse.

Les conditions méthodologiques requises pour permettre une voie d'accès à l'inconscient sexuel sont tributaires de sa définition et de la genèse qu'on lui attribue. La théorie de la séduction généralisée situe l'origine de l'inconscient sexuel de l'enfant dans l'autre, c'est à dire dans la sexualité infantile de l'adulte qui prend soin de lui. Laplanche estime que, pour comprendre les rapports entre théorie et pratique en psychanalyse, il ne faut pas se demander comment la théorie influence la pratique, mais reconnaître que la pratique a d'abord rendu la théorie possible. La cure, chez Freud, est dans une position première par rapport à la métapsychologie: l'invention de la pratique analytique est allée de pair avec la théorie de la séduction (Laplanche, 1999).

Laplanche considère en effet que, si le travail analytique est susceptible de modifier les forces en présence dans le conflit psychique, c'est parce qu'elles remettent en place les conditions d'une situation anthropologique fondamentale et universelle, dans laquelle l'enfant est en présence d'un adulte "qui signifie ce qu'il ne sait pas". (Laplanche, 1997, p. 332) Les conditions de possibilités du travail analytique reposent donc, d'une part, sur une situation - la situation analytique - et, d'autre part, sur une méthode - la méthode analytique.

1) La situation dont il est question est une situation de dissymétrie radicale. Selon Laplanche, la dissymétrie analytique ne se justifie que par la dissymétrie qui caractérise la position originaire de l'enfant. Il ne s'agit pas d'une dissymétrie qui proviendrait de son état de désaide ou de dépendance totale à l'égard de l'adulte sur le plan de l'autoconservation, de la tendresse et de l'attachement. Il s'agit de la dissymétrie qui découle du fait que l'enfant, à l'origine dépourvu d'inconscient, est en présence d'un adulte - ou d'un enfant plus âgé -, qui lui, est doté d'un tel inconscient et qui, dans cette position, adresse à l'enfant des messages compromis par son propre inconscient sexuel. Dans la théorie de la séduction généralisée, cette dissymétrie constitue le moteur du refoulement originaire et de la formation de l'inconscient sexuel de l'enfant. Rappelons que, dans cette théorie, le sexuel de l'enfant se forme à partir d'un matériau qui est déjà sexuel, en l'occurrence, la sexualité infantile de l'adulte qui prend soin de lui.

2) La méthode, quant à elle, implique un processus de détraduction/traduction, mis en mouvement par la situation analytique. Cette dernière se caractérise, d'une 
part, par le refusement (Versagung) de l'analyste, par son attention flottante et par sa bienveillance et, d'autre part, par la méthode associative/dissociative et par le travail de détraduction/traduction qu'elle met en œuvre. La méthode est indissociable de la situation: celle-ci sert à maintenir l'énigme du message qui provoque la tentative de traduction. Le refusement (Versagung) dont il est question ici va au-delà de la neutralité ou du refus de conseils. Il témoigne du respect profond de l'analyste envers son propre inconscient, sa propre altérité interne et ses limites, ce qui lui impose de se départir le plus possible de toute visée de maîtrise de l'analysant.

Le refusement implique aussi l'exigence de se tenir à distance de la synthèse, pour laisser autant que faire se peut le moi de l'analysant opérer lui-même les nouvelles traductions que le processus rend possible. Selon Laplanche (2007), la distance de l'analyste envers l'activité de synthèse constitue le véritable "test de délimitation essentielle entre psychanalyse et psychothérapie" (p. 271).

La correspondance que Laplanche (1997) établit entre la situation analytique et "ce qui se trouve à l'origine dans l'existence humaine" (p. 228) révèle deux des postulats épistémologiques dans lesquels s'inscrit sa théorie:

1. La théorie psychanalytique est aussi une anthropologie: elle recherche des catégories universelles de "l'être humain et du devenirhumain" (Laplanche, 1997, p. 232).

2. L'objet de la psychanalyse existe indépendamment de la psychanalyse: cet objet est donc, de ce fait, indépendant de la méthode, mais la méthode, elle, est orientée par l'objet (Laplanche, 1997).

La conception de la situation analytique comme une situation qui met en place des conditions permettant la réouverture de la situation anthropologique fondamentale scelle la continuité entre la clinique psychanalytique et les contributions de la psychanalyse en tant qu'anthropologie. Cette continuité permet de rendre compte du fait que la réalité de ce dont s'occupe la psychanalyse existe et agit dans tous les champs de l'activité humaine, Y compris dans les rapports des psychanalystes à la théorie psychanalytique et aux institutions psychanalytiques.

\section{QUELQUES ASPECTS DES THÉORIES ACTUELLES SUR LA PRATIQUE EN PSYCHANALYSE}

Les liens que nous venons de décrire entre métapsychologie et pratique psychanalytique éclairent aussi les rapports entre métapsychologie et psychothérapie. La théorie de Laplanche délimite de façon stricte le champ d'investigation de la psychanalyse par le découpage sexualité /autoconservation. Un tel découpage définit du même coup le champ d'action des psychothérapies analytiques et leur rôle dans la pratique. Il permet aussi de voir à quelles conditions le cadre de la situation thérapeutique favorise ou non l'accès aux dérivés de l'inconscient. Par ailleurs, il propose un modèle dont découle un travail plus éclairé en psychothérapie, notamment dans les situations où ce mode d'intervention s'avère plus adéquat.

Cependant, ce cadre théorique remet en question le pluralisme qui existe aujourd'hui dans la théorie psychanalytique. Au risque d'adopter ici une position polémique, je soutiendrai que, en plaçant la méthode psychanalytique à l'avant plan de l'élaboration de la métapsychologie, la théorie de Laplanche remet en question, les rapports généralement établis entre théorie et pratique en psychanalyse.

A ce sujet, je laisserai de côté les interprétations liées à la psychanalyse dite classique qui, malgré un cadre où les interventions de l'analyste pouvaient être parcimonieusement distribuées, n'en introduisaient pas moins un mouvement de synthèse gravitant autour de la phylogénèse et des conceptions mythosymboliques de l'inconscient (par exemple, l'Oedipe, la castration, les fantasmes originaires, la scène primitive, la castration). Un tel mouvement est inhérent à l'introduction directe de la métapsychologie dans l'interprétation des contenus de l'inconscient. En effet, utiliser la métapsychologie comme grille interprétative des contenus conduit nécessairement à une liaison. De plus, cette utilisation implique l'introduction d'un code de traduction. Laplanche (1999) a souvent dénoncé les embuches que comportent les théories mythosymboliques et phylogénétiques de l'inconscient pour le travail analytique. Sa formule: "Silence aux associations, moi, la castration, je parle!" (p. 282) résume bien comment le recours à la théorie - théorie au demeurant critiquable au plan métapsychologique - peut agir de façon intrusive comme frein au travail spécifiquement analytique. "Là où la symbolique parle, c'est quand l'association libre se tait" (Laplanche, 1999, p. 280).

Sans insister davantage sur les problèmes liés à métapsychologie classique en psychanalyse, nous porterons maintenant notre attention sur les écoles contemporaines, notamment sur les courants aujourd'hui dominants dans la psychanalyse anglosaxonne, qui représentent par la même occasion les courants dominants dans la psychanalyse internationale. Ces courants, découlent, soit des écoles de relations d'objet, soit des orientations relationnelles ou intersubjectivistes, soit d'un alliage de ceux-ci. Ils 
adoptent des formes variées qui ne s'excluent pas l'une l'autre. En voici quelques unes: intersubjectivité, conception narrative du travail analytique impliquant la co-construction d'une nouvelle réalité, conception $\mathrm{du}$ travail analytique en tant qu'acquisition de nouveaux modes relationnels, intégration des théories de l'attachement dans le corpus théorique de la psychanalyse, établissement d'un pont entre psychanalyse et neuro-sciences.

Vous me direz que ma description inclut la plupart des théories contemporaines de la pratique analytique. On peut en effet se demander si une grande partie des psychanalyses ne s'assimile pas de plus en plus à des psychothérapies pures et simples. La distinction métapsychologique entre psychanalyse et psychothérapie subsiste difficilement dans la pratique quand les deux critères - qui, selon Laplanche, constituent les conditions de possibilité du travail analytique - au sens strict - en sont absents. Cette affirmation ne constitue pas une critique de l'effectivité de ces approches, ni de leur à propos en tant que pratiques thérapeutiques. Il est cependant essentiel de soulever ce problème pour éviter que la discussion sur la nécessité d'un choix métapsychologique en psychanalyse ne soit totalement inutile, ce qui serait le cas si dans la celle-ci n'était plus reliées à une pratique spécifique. Par ailleurs si on voulait débattre des liens entre théorie et pratique en psychothérapie, il faudrait se référer à des catégories différentes.

Pour illustrer le problème des frontières poreuses entre travail analytique et le travail psychothérapeutique dans les écoles relationnelles contemporaines, je voudrais prendre deux exemples: celui de l'usage de l'intersubjectivité en psychanalyse, et la théorie épistémologique de Fonagy sur les liens entre théorie et pratique.

\section{La notion d'intersubjectivité en psychanalyse}

Les écoles relationnelles rejettent l'idée et la réalité d'une dissymétrie dans la situation analytique ${ }^{3}$. Elles définissent l'inter/subjectivité comme l'action mutuelle des subjectivités en présence l'une de l'autre et comme ce qui, corrélativement, délimite le champ dans lequel se développe la subjectivité Ce modèle relationnel de la constitution de la subjectivité, dans lequel le nous précède le moi, est emprunté à la phénoménologie. Il n'est pas sans poser problème au plan philosophique. En effet, si la subjectivité ne peut naître que de l'intersubjectivité, d'où proviennent les deux sujets susceptibles de former ensemble le premier rapport intersubjectif?

3 Sauf sous l'angle de la recherche de l'aide, établissant alors un parallèle à la situation parent/enfant.
Par ailleurs, en psychanalyse, la position de l'école intersubjectiviste est tout aussi problématique. La subjectivité ne constitue pas un concept psychanalytique. Au contraire, la psychanalyse est née de l'éclatement de la notion de sujet. Elle est issue de la constatation que le moi était, en grande partie, agi par des forces qui lui sont étrangères. Les représentants du courant intersubjectiviste ne semblent pas s'interroger sur ce point. Ils démontrent peu d'intérêt pour la localisation topique de la subjectivité, ni pour la définition métapsychologique du sujet qui en serait porteur. Leur centre d'attention n'est pas la pulsion, mais l'affect. En évoquant la rencontre de deux subjectivités, soit au moment de la formation de la vie psychique, soit, comme moteur du changement dans la cure analytique, le courant intersubjectiviste se situe dans un cadre de référence psychologique et non plus métapsychologique. Il n'est donc pas surprenant que l'instance à laquelle se réfère la plupart du temps la clinique anglo-saxonne soit le self, qui, en termes freudiens correspond au versant identificatoire et métaphorique du mois.

En ce qui a trait à la méthode, les courants intersubjectivistes ont délaissé la notion de libre association au profit d'une technique interprétative fondée sur l'attribution de sens et sur la communication interpersonnelle. Ils estiment que l'analyse doit servir à donner une signification à l'expérience vécue, signification qui doit être ressentie comme authentique tant par l'analyste que par l'analysant. Dans ce contexte, l'interprétation n'a pas nécessairement à prendre appui sur les associations du patient mais elle s'élabore plutôt à partir du sens attribué, dans le cadre de la relation, aux réactions affectives des deux parties en présence et, peut-être surtout, à celles de l'analyste. Elle constitue dès lors une activité de communication interpersonnelle qui procure une occasion de contacts affectifs.

L'importance que les écoles intersubjectivistes accordent à la co-création d'une nouvelle expérience subjective accrédite la thèse voulant que le travail qu'elles favorisent est un travail psychothérapeutique. Celui-ci s'effectue en effet au plan de l'autohistorisation et de l'auto-construction du sujet ainsi que de l'élaboration d'une narrativité plus riche. En raison des moyens méthodologiques que ces écoles mettent en place, les fondements inconscients des conduites et des pensées de l'analysant ont peu de chance de ressurgir. Comme le souligne Laplanche (2007), les résultats de ce travail ne sont pas négligeables, mais, "pour parler en termes de traduction, on traduit à partir d'une traduction déjà existante, avec peu de référence au texte original" (p. 272). 
Par ailleurs, la notion de subjectivité à laquelle se réfèrent les écoles relationnelles présente plusieurs traits fonctionnalistes, qui l'inscrivent dans la mouvance pragmatiste des philosophies de l'esprit. Pour ces écoles, comme pour les philosophies de l'esprit, la subjectivité s'assimile au ressenti dont on prend conscience ( le stream of consciousness, de James). La conscience se définit alors par sa fonction, en l'occurrence la connaissance. Une telle position témoigne de l'équivalence posée par les écoles relationnelles entre la subjectivité dans le cadre de la cure et la subjectivité psychologique, notamment dans le rapport que celle-ci entretient avec la connaissance et la projection. Comme les écoles intersubjectivistes, tel que nous l'avons déjà dit, se préoccupent peu de la genèse de la subjectivité en tant que phénomène de l'âme, elles lui attribuent volontiers une origine qui sort du champ spécifique de la psychanalyse, origine dont le substrat serait biologique ou neurophysiologique. Elles constituent de ce fait un terreau propice à l'intégration, en psychanalyse, de modèles propres aux théories de l'attachement, aux sciences cognitives et aux neurosciences (Tessier, 2005).

\section{La position épistémologique de Fonagy}

L'affiliation avec le pragmatisme que l'on retrouve dans les courants relationnels et intersubjectivistes ainsi que leurs liens avec les philosophies de l'esprit (philosophy of mind), nous conduisent à examiner la position exposée par Fonagy (2003) dans Some Complexities in the Relationship of Psychoanalytic Theory to Technique. Cette position aborde de façon directe la question qui nous occupe et, ce qui est intéressant pour nous, elle repose sur des fondements philosophiques qui se situent à l'opposé de ceux auxquels se rattachent la théorie de Laplanche. Il s'agit aussi d'une théorie que l'on retrouve, à divers degrés, dans plusieurs conceptions contemporaines de la pratique analytique, même dans des courants qui ne se réclament de l'orientation de Fonagy (2003).

Fonagy (2003) affirme en effet explicitement que la psychanalyse doit être comprise dans le cadre du pragmatisme et, ce faisant, il la réinterprète en fonction des philosophies de l'esprit. Je soulignerai quelques points qui apparaissent particulièrement éloquents à cet égard:

a. Dans le texte de Fonagy (2003), la psychanalyse est conçue comme une théorie de l'esprit (a theory of mind) et non une théorie de l'âme (Seele, soul).

Les autres points que j'exposerai maintenant permettent de situer sa conception de l'inconscient au plan épistémologique: b. Il existe des preuves qui valident les théories à l'effet que l'esprit comporte des éléments inconscients. En effet, les neurosciences démontrent que la plus grande partie du fonctionnement du cerveau est inconscient. (Mon commentaire: Dans ce contexte, le terme inconscient ne peut que renvoyer à un inconscient descriptif, en l'occurrence, à ce qui est hors de la conscience ou à ce dont on n'a pas conscience (non awareness). Comme l'écrit Fonagy (2003), rendre l'inconscient conscient signifie colmater les brèches (fill the gaps) qui demeurent dans la façon dont le patient comprend sa vie).

c. La théorie psychanalytique provient de connaissances inconscientes, c'est à dire intuitives, et de métaphores scientifiques. La théorie psychanalytique correspond en réalité à une psychologie du sens commun, approfondie par l'expérience clinique.

d. La découverte de la psychanalyse freudienne correspond en fait aux principes de la mentalisation: elle a, d'une part, reconnu que l'intentionnalité n'était pas restreinte à la conscience et d'autre part, que l'accroissement de la capacité de penser, pour y de inclure les désirs, les émotions et les idées dont nous n'étions pas conscients, est thérapeutique si elle se produit dans le cadre d'une relation d'attachement.

e. Le domaine de la théorie psychanalytique est en premier lieu la psychopathologie. La psychanalyse permet aussi d'éclairer d'autres aspects du comportement humain, tels que la littérature et les arts.

f. Les psychopathologies constituent des états mentaux, en d'autres termes, des désirs et des croyances, vécus de façon inconsciente;

g. La pratique doit être libérée de la théorie. La théorie n'a de valeur que dans la mesure où elle peut servir à élaborer le sens d'un comportement dans des termes qui sont communicables au patient.

Comme il le reconnaît explicitement, Fonagy (2003) se situe dans la tradition du pragmatisme nordaméricain, tradition qui se situe à la source de l'épistémologie de l'action qu'il préconise dans sa description de la fonction de la théorie en psychanalyse: "The North American tradition of pragmatism lies at the root of this postmodern action based epistemology" (Fonagy, 2003, p. 37). La position de Fonagy (2003) est historiquement liée à l'évolution et à la remise en question du behaviorisme, qui excluaient les croyances, les désirs et l'intentionnalité de l'analyse des déterminants du 
comportement. Les philosophies de l'esprit (Dennet \& Fodor, cité par Fisette \& Poirier, 2002.) ont réhabilité ces éléments en tant qu'élément constitutifs d'une théorie de l'esprit, théorie sur laquelle s'appuient les positions de Fonagy en psychanalyse. C'est pourquoi le modèle de l'apprentissage comme facteur de transformation thérapeutique, y occupe une place centrale. Il s'agit particulièrement de l'apprentissage dans la sphère affective, par l'exposition à de nouveaux modes relationnels, expérience qui serait favorable au développement d'un nouveau réseau de croyances et de représentations permettant au patient de mieux rendre compte de son propre comportement et du comportement d'autrui.

Laplanche (2007) se rattache au contraire à la tradition rationaliste. Il insiste sur la réalité de l'inconscient sexuel en tant que partie constituante l'âme, indépendamment de la conscience subjective qu'on peut en acquérir. Pour Laplanche, l'inconscient n'est pas constitué de désirs, d'émotions, ou de croyances dont nous ne rendons pas compte. Les contenus de l'inconscient possèdent au contraire la réalité d'une chose. Par leurs caractéristiques, ils échappent aux catégories humaines. Ils sont étrangers au sens, de même qu'à toute logique de communication. Ils sont irréductibles à une psychologie des besoins et de la motivation.

Par ailleurs, Laplanche insiste sur la nécessité d'affirmer l'origine essentiellement humaine de ces contenus inconscients devenus signifiants désignifiés. Il postule qu'on doit pouvoir en rendre compte au moyen de la raison discursive et dans une perspective historique. Pour cette raison, la théorie de la séduction généralisée ne quitte pas le champ épistémologique de la psychanalyse dans sa description de l'origine de l'inconscient sexuel Autrement dit, cette théorie refuse d'attribuer l'origine de l'inconscient à la biologie, à l'anatomie ou à un usage mythique de celles-ci. Elle situe la frontière de la psychanalyse aux confins de ce qui relève de l'ordre du vivant, en l'occurrence l'autoconservation, y compris l'attachement, et de ce qui initie le processus d'humanisation, en l'occurrence, le refoulement originaire et la formation de l'inconscient sexuel, qui envahit par la suite l'ensemble des activités humaines.

La position de Laplanche ne signifie pas que les autres disciplines, notamment, la biologie, la neurophysiologie, la psychologie, - y compris l'observation des nourrissons - n'ont rien à apporter à la compréhension de la vie psychique. Elle vise au contraire à mieux délimiter le champ épistémologique de la psychanalyse, pour mieux bénéficier des contributions respectives des autres disciplines.

Malgré leurs différences, les positions de Laplanche et de Fonagy se recoupent sur un point. Les deux soutiennent que les théories métapsychologiques ne peuvent servir à expliquer ou à organiser le contenu des observations cliniques. Leurs raisons sont cependant différentes. Pour Fonagy (2003), il s'agit d'éliminer les aspects de la théorie qui ne sont pas utiles à la pratique et d'utiliser la théorie psychologique comme aide subjective pour compléter les schèmes narratifs du patient. Pour Laplanche (1999), la métapsychologie sert à rendre compte du mode d'action du travail analytique. La théorie doit cependant toujours être tenue à distance de la cure pour laisser libre cours aux associations de l'analysant. Soulignons aussi que, dans la théorie de Laplanche, seule la situation anthropologique fondamentale est universelle. Les contenus de l'inconscient, tout en n'étant pas subjectifs - ils proviennent de l'autre et agissent comme une force étrangère au sujet -, sont singuliers et tributaires du refoulement individuel. La métapsychologie de Laplanche ne pourrait donc pas servir ni de code, ni d'aide à la traduction dans la cure.

\section{CONCLUSION}

Je voudrais maintenant conclure en revenant aux thèmes de la nécessité d'un choix parmi les différentes écoles en psychanalyse, en lien avec les considérations que j'ai énoncées plus tôt sur la visée d'émancipation.

Écrivait Marx (1845/1994): "Les philosophes n'ont fait qu'interpréter le monde de diverses manières; ce qui importe est de le transformer" (p. 235). Comment l'interprétation peut-elle mener à la transformation? Cette question est au coeur de notre débat. Elle soulève à son tour un ensemble d'interrogations: Toutes les façons d'interpréter la réalité se valent-elles? Les interprétations du monde présentent-elles toutes le même rapport à la pratique? Toutes ont-elles un impact sur la pratique?

On sait que Marx pensait que certaines conditions étaient nécessaires pour qu'une théorie ait un effet transformateur. L'exigence d'un rapport historique et dialectique à la réalité figure en première place parmi celles-ci. La reconnaissance d'une réalité qui, non seulement nous résiste, mais qui existe indépendamment de notre conscience, constitue un postulat central de la tradition rationaliste. En psychanalyse, cette réalité s'incarne sous la forme de l'inconscient.

La visée d'émancipation de la psychanalyse me paraît indissociable de la reconnaissance du réalisme de l'inconscient et de sa réalité, en tant que réalité qui nous aliène. Il s'agit de reconnaître la dimension de l'âme qui se présente à nous comme une chose interne, délestée de ses caractéristiques humaines, qui résiste au sens et à la communication et qui 
constitue, en partie du moins, le matériau et la cause de nos pensées et de nos actions. La métapsychologie doit nous permettre de comprendre par quelles exigences méthodologiques nous pouvons avoir accès à cette réalité afin que, dans des circonstances favorables le rapport entre les forces en présence puisse se modifier dans le sens d'une plus grande liberté. Un tel objectif nécessite que la théorie puisse aussi rendre compte de la formation de l'inconscient sexuel et de son lien avec le processus d'humanisation, y compris celui qu'il entretient avec la formation du moi et de la réflexivité.

Comme je l'ai déjà répété plusieurs fois, une telle position ne discrédite pas le travail psychothérapeutique qui fait partie intégrante de la cure analytique, ni celui des thérapies d'inspiration analytique. La reconnaissance de la réalité de l'inconscient sexuel permet de situer au plan topique le travail en cours dans les moments psychothérapeutiques de l'analyse ou dans la psychothérapie. Elle permet aussi de conserver un recul nécessaire face aux théories explicatives et normatives qui, dans ces situations, viendraient trop vite substituer la synthèse proposée par l'analyste aux remaniements d'autohistorisation qui proviendraient de l'analysant.

Une théorie métapsychologique n'est pas un instrument qui sert à interpréter et à construire. Il s'agit d'un outil pour comprendre et non pas pour agir (Laplanche, 2007). Les théories en psychanalyse ne sont pas équivalentes. Elles reposent sur des présupposés philosophiques reliés à des conceptions différentes de l'autonomie humaine. Les choix épistémologiques comportent en conséquence une dimension éthique, voire morale. Le subjectivisme en psychanalyse, malgré son caractère en apparence libéral, comporte le danger de l'empirisme qu'avait déjà décrit Schiller (in Goethe-Schiller, 1994): celui de se soustraire à la catégorie de l'erreur et, de ce fait, d'échapper à l'exigence de vérité.

\section{REFERÊNCIAS}

Fisette, D. \& Poirier, P. (2002). Philosophie de l'esprit. Psychologie du sens commun et sciences de l'esprit. Paris: Vrin.

Fonagy, P. (2003). Some Complexities in the Relationship of Psychoanalytic Theory to Technique. Psychoanalytic Quarterly, 72:13-47.

Fonagy, P. (2006) The failure of practice to inform theory and the role of implicit theory in bridging the transmission gap. (p. 69-86) In J. Canestri (ed.) Psychoanalysis from practice to theory. London: Whurr.

Freud, S. (1981). Le moi et le ça. Traduction sous la responsabilité de A. Bourguignon. Payot: Paris. (Original publié en 1923).

Freud, S. (1992). L'interprétation des rêves. Paris: PUF. (Original publié en 1900).

Freud, S. (1998). Analyse avec fin et l'analyse sans fin, Résultats idées, problèmes II, (pp. 231-268). Paris: PUF. (Original publié en 1937).

Goethe-Schiller. (1994) Correspondance 1794- 1805. Trad. L. Herr. Paris: Gallimard.

Laplanche, J. (2007). Sexual: La sexualité élargie au sens freudien. Paris: PUF. Coll Quadrige.

Laplanche, J. (1997). Le primat de l'autre en psychanalyse. Paris. Flammarion, Coll. Champs.

Laplanche, J. (1999). Entre séduction et inspiration: l'homme. Paris: PUF. Coll. Quadrige.

Laplanche, J. (2006). Problématiques VII: Le fourvoiement biologisant de la sexualité chez Freud.. Paris: PUF. Coll. Quadrige.

Marx, K. (1994). Thèses sur Feurbach, thèse XI. In K. Marx. Philosophie (pp. 232-235). Paris: Gallimard. Coll. Folio. (Original publié en 1845).

Tessier, H. (2005). La psychanalyse américaine. Paris: PUF. Coll Que sais-je?

Wallerstein, R. (1988). One Psychoanalysis or many? International Journal of psychoanalysis, 69:5-21.

Recebido em 19-07-2012

Aceito em 15-12-2012 